\title{
Cytotoxic Sesquilignans from the Roots of Saururus chinensis
}

\author{
Young-Won Chin, Xing-Fu Cai, Kyung-Seop Ahn, Hyeong-Kyu Lee, and Sei-Ryang Oh* \\ Bio-Therapeutics Research Institute, Korea Research Institute of Bioscience and Biotechnology, Ochangeup, \\ Cheongwongun, ChungBuk 363-883, Korea. *E-mail: seiryang@kribb.re.kr \\ Received April 26, 2010, Accepted May 25, 2010
}

Key Words: Saururus chinensis, Sesquilignans, Cytotoxicity, Saururaceae

Saururus chinensis Hort. ex Loudon (Saururaceae) is a perennial herb distributed in China and Korea, and has been used as a folk medicine for the treatment of edema, gonorrhea, jaundice, pneumonia, and several inflammatory diseases in Korea. ${ }^{1}$ Previous studies of $S$. chinensis reported the occurrence of lignans, ${ }^{2-9}$ aristolactams, ${ }^{10}$ flavonoids, ${ }^{11}$ and furanoditerpenes, ${ }^{12}$ and a wide range of biological activities including antioxidant activity, ${ }^{5}$ hepatoprotective activity, ${ }^{6}$ cytotoxic activity, ${ }^{13-18}$ antiinflammatory activity, ${ }^{19-22}$ anti-atherogenic activity, ${ }^{21,23}$ and immunosuppressive activity. ${ }^{24}$ This paper reports the structures elucidation of the two new lignans and six known compounds, along with their cytotoxicity.

Compound 1 was obtained as a colorless powder and its molecular formula was determined to be $\mathrm{C}_{31} \mathrm{H}_{38} \mathrm{O}_{8}$, based on the [M-H] ${ }^{-}$peak at $m / z 537.2471$ (calcd 537.2488) in the HRESIMS. The ${ }^{1} \mathrm{H}-\mathrm{NMR}$ spectroscopic data of compound $\mathbf{1}$ indicated the presence of a tetrahydrofuran-type lignan unit, as judged from the signals for two methines at $\delta_{\mathrm{H}} 2.34$ ( $\mathrm{H}-8$ and $\left.\mathrm{H}-8^{\prime}\right)$, for oxymethines at $\delta_{\mathrm{H}} 5.65\left(\mathrm{H}-7\right.$ and $\left.\mathrm{H}-7^{\prime}\right)$, and for methyl groups at $\delta_{\mathrm{H}} 0.78(\mathrm{H}-9)$ and $0.74\left(\mathrm{H}_{-} 9^{\prime}\right)$, as well as the signals at $\delta_{\mathrm{H}}$ 7.20 (d, $J=1.7 \mathrm{~Hz}, \mathrm{H}-2), 7.30(J=8.2 \mathrm{~Hz}, \mathrm{H}-5), 7.36$ (br d, $J=$ $8.2 \mathrm{~Hz}, \mathrm{H}-6), 7.19$ (d, $\left.J=1.7 \mathrm{~Hz}, \mathrm{H}-2^{\prime}\right), 7.34$ (d, $J=8.2 \mathrm{~Hz}$, H-5'), and 7.09 (dd, $\left.J=8.2,1.7 \mathrm{~Hz}, \mathrm{H}-6^{\prime}\right)$ corresponding to two 1,3,4-trisubstitued benzene rings. ${ }^{4,25}$ An additional phenylpropanoid unit was observed in compound 1 from the proton signals for two oxymethines at $\delta_{\mathrm{H}} 4.95$ (H-8"), and $5.42(\mathrm{H}-7 ")$, for a methyl at $\delta_{\mathrm{H}} 1.60$ (H-9") and for a 1,3,4-trisubstituted benzene ring at $\delta_{\mathrm{H}} 7.58\left(\mathrm{~d}, J=1.6 \mathrm{~Hz}, \mathrm{H}-2^{\prime \prime}\right), 7.01(\mathrm{~d}, J=8.2$ $\left.\mathrm{Hz}, \mathrm{H}-5^{\prime \prime}\right)$, and 7.07 (dd, $\left.J=8.2,1.6 \mathrm{~Hz}, \mathrm{H}-6^{\prime \prime}\right){ }^{4}$ The location of the phenylpropanoid group was confirmed by the HMBC correlation between $\mathrm{H}-8$ " $\left(\delta_{\mathrm{H}} 4.95\right)$ and $\mathrm{C}-4$ ' $\left(\delta_{\mathrm{C}} 147.5\right)$, suggesting this phenylpropanoid is attached to $\mathrm{C}-4$ ' on the tetrahydrofuran-type lignan moiety through an ether linkage. The relative stereochemistry of the tetrahydrofuran ring in compound 1 was established by the observed NOE correlations of H-9 with H-8', $\mathrm{H}-2$, and $\mathrm{H}-6$ as well as $\mathrm{H}-9^{\prime}$ with $\mathrm{H}-8, \mathrm{H}-2^{\prime}$, and $\mathrm{H}-6$ ', indicating the 7,8-cis-8, $8^{\prime}$-trans-7', $8^{\prime}$-cis configuration. ${ }^{4,26}$ In addition, the chemical shifts of C-9" $\left(\delta_{\mathrm{C}} 15.3\right), \mathrm{C}-7$ " $\left(\delta_{\mathrm{C}} 75.7\right)$, and C-8" $\left(\delta_{\mathrm{C}}\right.$ $81.1)$, and the coupling constant $(J=3.4 \mathrm{~Hz})$ of $\mathrm{H}-7$ " was supportive of the relative configuration of C-7" and C-8" as being erythro. ${ }^{4,26}$ Futhermore, the positive Cotton effect at $231 \mathrm{~nm}$ enabled to assign the configuration of C-7" and 8 " as $R$ and $S$, respectively. ${ }^{27}$ Thus, the structure of compound 1 was determined as 7"R, 8 " $S$-saucerneol, a diastereomer of (-)-saucerneol (3).

Saucerneol J (2) had a molecular formula $\left(\mathrm{C}_{30} \mathrm{H}_{36} \mathrm{O}_{8}\right)$ and exhibited a close resemblance to compound 1 in their ${ }^{1} \mathrm{H}$ and ${ }^{13} \mathrm{C}$ NMR spectroscopic data except for the presence of three methoxy groups. There were differences in the chemical shifts and coupling constants of H-7' in compounds $2\left(\delta_{\mathrm{H}} 4.54, J=9.3\right.$ $\mathrm{Hz}$ ) and $1\left(\delta_{\mathrm{H}} 5.65, J=6.4 \mathrm{~Hz}\right)$, indicating the opposite configuration at C-7' position in compound $\mathbf{2}$ compared to compound $\mathbf{1}$.

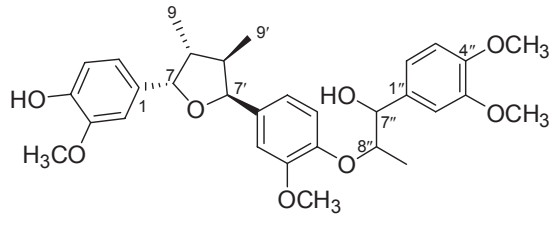

7",8"

1 erythro<smiles>COc1cc(C(O)C(C)Oc2ccc([C@@H]3O[C@@H](c4ccc(O)c(OC)c4)[C@H](C)[C@H]3C)cc2OC)ccc1O</smiles>

7",8"

2 erythro

Figure 1. Structures of compounds 1-2.


Figure 2. Key ${ }^{1} \mathrm{H}^{1}{ }^{1} \mathrm{H}$ COSY $(\boldsymbol{-})$ and $\mathrm{HMBC}(\mathrm{H} \longrightarrow \mathrm{C})$ and NOESY $(\longleftrightarrow)$ correlations of compound $\mathbf{1}$. 
Table 1. ${ }^{1} \mathrm{H}$-and ${ }^{13} \mathrm{C}-\mathrm{NMR}$ data $(\delta)$ of $\mathbf{1 - 2}\left(\text { Pyridine- } d_{5}\right)^{a}$

\begin{tabular}{|c|c|c|c|c|}
\hline \multirow{2}{*}{ position } & \multicolumn{2}{|c|}{1} & \multicolumn{2}{|c|}{2} \\
\hline & $\delta_{\mathrm{H}}$ & $\delta_{\mathrm{C}}$ & $\delta_{\mathrm{H}}$ & $\delta_{\mathrm{C}}$ \\
\hline 1 & - & $136.5(\mathrm{~s})$ & - & $135.6(\mathrm{~s})$ \\
\hline 2 & $7.20 \mathrm{~d}, 1.7$ & $111.6(d)$ & $7.25 \mathrm{~d}, 1.7$ & $112.1(\mathrm{~d})$ \\
\hline 3 & - & $151.5(\mathrm{~s})$ & - & $151.5(\mathrm{~s})$ \\
\hline 4 & - & $147.6(\mathrm{~s})$ & - & $147.8(\mathrm{~s})$ \\
\hline 5 & $7.30 \mathrm{~d}, 8.2$ & $117.5(\mathrm{~d})$ & $7.28 \mathrm{~d}, 8.1$ & $116.5(d)$ \\
\hline 6 & 7.36 br d, 8.2 & $120.1(\mathrm{~d})$ & 7.31 br d, 8.1 & $120.6(d)$ \\
\hline 7 & $5.65 \mathrm{~d}, 6.4$ & $84.6(d)$ & $5.30 \mathrm{~d}, 8.6$ & 82.7 (d) \\
\hline 8 & $2.34 \mathrm{ddq}, 12.8,6.4,6.4$ & $44.5(d)$ & $2.24 \mathrm{ddq}, 8.6,7.0,6.5$ & 45.7 (d) \\
\hline 9 & $0.78 \mathrm{~d}, 6.4$ & $15.3(\mathrm{q})$ & $0.73 \mathrm{~d}, 7.0$ & $14.9(\mathrm{q})$ \\
\hline $1^{\prime}$ & - & $133.8(\mathrm{~s})$ & - & $133.2(\mathrm{~s})$ \\
\hline $2^{\prime}$ & $7.19 \mathrm{~d}, 1.7$ & $112.2(\mathrm{~d})$ & $7.42 \mathrm{~d}, 1.7$ & $111.2(\mathrm{~d})$ \\
\hline $3^{\prime}$ & - & $148.9(\mathrm{~s})$ & - & $149.2(\mathrm{~s})$ \\
\hline $4^{\prime}$ & - & $147.5(\mathrm{~s})$ & - & $148.5(\mathrm{~s})$ \\
\hline $5^{\prime}$ & $7.34 \mathrm{~d}, 8.2$ & $116.6(d)$ & $7.30 \mathrm{~d}, 8.1$ & $116.1(\mathrm{~d})$ \\
\hline $6^{\prime}$ & $7.09 \mathrm{dd}, 8.2,1.7$ & $119.8(d)$ & $7.32 \mathrm{dd}, 8.1,1.7$ & $120.5(d)$ \\
\hline $7^{\prime}$ & $5.65 \mathrm{~d}, 6.4$ & $84.3(\mathrm{~d})$ & $4.54 \mathrm{~d}, 9.3$ & $87.4(\mathrm{~d})$ \\
\hline $8^{\prime}$ & $2.34 \mathrm{ddq}, 12.8,6.4,6.4$ & $44.5(\mathrm{~d})$ & $1.93 \mathrm{ddq}, 9.3,6.5,6.5$ & 47.9 (d) \\
\hline $9^{\prime}$ & $0.74 \mathrm{~d}, 6.4$ & $15.3(\mathrm{q})$ & $1.05 \mathrm{~d}, 6.5$ & $14.5(q)$ \\
\hline $1 "$ & - & $136.8(\mathrm{~s})$ & - & $135.1(\mathrm{~s})$ \\
\hline $2^{\prime \prime}$ & $7.58 \mathrm{~d}, 1.6$ & $112.2(\mathrm{~d})$ & $7.59 \mathrm{~d}, 1.6$ & $111.3(\mathrm{~d})$ \\
\hline $3 "$ & - & $150.2(\mathrm{~s})$ & - & $148.9(\mathrm{~s})$ \\
\hline $4 "$ & - & $149.5(\mathrm{~s})$ & - & $147.7(\mathrm{~s})$ \\
\hline $5^{\prime \prime}$ & $7.01 \mathrm{~d}, 8.2$ & $112.6(d)$ & $7.31 \mathrm{~d}, 8.1$ & $115.8(\mathrm{~d})$ \\
\hline $6 "$ & $7.07 \mathrm{dd}, 8.2,1.6$ & $120.3(d)$ & $7.29 \mathrm{dd}, 8.1,1.6$ & 120.7 (d) \\
\hline $7 "$ & $5.42 \mathrm{~d}, 3.4$ & $75.7(d)$ & $5.42 \mathrm{~d}, 3.9$ & 74.8 (d) \\
\hline $8^{\prime \prime}$ & $4.95 \mathrm{dq}, 6.2,3.4$ & $81.1(\mathrm{~d})$ & $4.90 \mathrm{dq}, 6.2,3.9$ & 80.3 (d) \\
\hline $9 "$ & $1.60 \mathrm{~d}, 6.2$ & $15.3(\mathrm{q})$ & $1.60 \mathrm{~d}, 6.2$ & $14.3(\mathrm{q})$ \\
\hline $3-\mathrm{OCH}_{3}$ & $3.84 \mathrm{~s}$ & $56.5(\mathrm{q})$ & $3.82 \mathrm{~s}$ & $56.4(q)$ \\
\hline $3^{\prime}-\mathrm{OCH}_{3}$ & $3.85 \mathrm{~s}$ & $56.5(\mathrm{q})$ & $3.86 \mathrm{~s}$ & $56.4(q)$ \\
\hline $3 "-\mathrm{OCH}_{3}$ & $3.82 \mathrm{~s}$ & $56.3(q)$ & $3.80 \mathrm{~s}$ & $56.3(\mathrm{q})$ \\
\hline 4"--OCH 3 & $3.77 \mathrm{~s}$ & $56.4(q)$ & - & - \\
\hline
\end{tabular}

${ }^{a}$ Assignments were based on ${ }^{1} \mathrm{H}-{ }^{1} \mathrm{H}$ COSY, DEPT, HMQC and HMBC spectra.

The CD spectroscopic data exhibited the positive Cotton effect at $232 \mathrm{~nm}$ in the same manner. Therefore, the structure of compound 2 was confirmed to be 7'-epi-7"R,8"S-4"-demethylsaucerneol.

The known compounds were in good agreement with previously reported NMR data and were consequently identified as (-)-saucerneol $(3)$ with a $[\alpha]_{\mathrm{D}}$ value of $-71.1(c 0.1 \mathrm{MeOH})$ $\left[\right.$ Lit. $\left.[\alpha]_{\mathrm{D}}-91.8\right]$, ${ }^{25}$ threo,erythro-manassantin A (4), ${ }^{16} 4-O-$ demehylmanassantin B (5), ${ }^{18}$ erythro,erythro-manassantin A (6), ${ }^{16}$ manassantin B (7) ${ }^{28}$ and manassantin A (8). ${ }^{17}$

All the compounds isolated were evaluated against HL-60 (human promyelocytic leukemia) cells. Compounds 1-8 exhibited cytotoxicity $\left(\mathrm{IC}_{50}, 0.5,7.1,3.3,5.2,3.6,2.3,8.5\right.$ and 0.8 $\mu \mathrm{M}$, respectively) against HL-60 cell lines (camptothecin, $\mathrm{IC}_{50}$ $0.8 \mu \mathrm{M})$.

\section{Experimental Section}

General experimental procedures. Melting points were determined on a Kofler micro-hotstage (uncorrected). Optical rota- tions were measured on a JASCO P-1020 polarimeter. UV spectra were measured on a Shimadzu UV-1601 UV-visible. CD spectroscopic data were obtained from JASCO-720 CD spectrometer. The NMR spectra were recorded on a Varian Unity 400 FT-NMR spectrometers with the tetramethylsilane as an internal standard. Chemical shifts are presented in ppm. HRESIMS were measured on a Waters Q-Tof Premier mass spectrometer. Column chromatography (CC) was performed on silica gel (70 - 230 and 230 - 400 mesh, Merck), reverse-phase C18 gel (40 $\mu \mathrm{m}$, Nacalai Inc., Japan). Thin layer chromatography (TLC) was performed on Kieselgel $60 \mathrm{~F}_{254}$ (Merck) or $\mathrm{RP}-18 \mathrm{~F}_{254}$ (Merck) plates. Spots were visualized by spraying $10 \%$ aqueous $\mathrm{H}_{2} \mathrm{SO}_{4}$ solution on the plates and heating them for $5 \mathrm{~min}$.

Plant material. The roots of Saururus chinensis was collected at Jeju (Korea) in July 2008 and dried at room temperature. A voucher specimen (00250) is deposited at the Plant Extract Bank, Korea Research Institute of Bioscience and Biotechnology, Daejeon, Korea.

Extraction and isolation. The dried roots of $S$. chinensis 
$(6.5 \mathrm{~kg})$ was extracted with $\mathrm{MeOH}$ at room temperature $(3 \times$ $20 \mathrm{~L}$ ) to obtain $0.65 \mathrm{~kg}$ of the solid extract. The $\mathrm{MeOH}$ extract was suspended in $\mathrm{H}_{2} \mathrm{O}$ and extracted with EtOAc $(3 \times 3 \mathrm{~L})$ to give the EtOAc-soluble fractions $(85 \mathrm{~g})$. The EtOAc-soluble fraction ( $84 \mathrm{~g}$ ) was chromatographed on a silica gel column eluted with a stepwise gradient of hexane and EtOAc to yield 14 fractions (fr. SC1-SC14). Fr. SC12 (1.2 g) was chromatographed on a RP C-18 column $\left(\mathrm{MeOH} / \mathrm{H}_{2} \mathrm{O}, 7: 3\right)$ to yield 19 sub-fractions (fr. SC12-1-SC12-19). Fr. SC12-15 (0.14 g) was chromatographed on a RP C-18 column $\left(\mathrm{MeOH} / \mathrm{H}_{2} \mathrm{O}, 2: 1\right)$ to give compounds $\mathbf{1}(13.2 \mathrm{mg})$ and $\mathbf{3}(98.1 \mathrm{mg})$. Fr. SC12-19 $(0.103 \mathrm{~g})$ was chromatographed on a RP C-18 column $(\mathrm{MeOH} /$ $\left.\mathrm{H}_{2} \mathrm{O}, 2: 1\right)$ to give compound 4 (17.8 mg). Fr. SC14 (0.88 g) was chromatographed on a RPC-18 column $\left(\mathrm{MeOH} / \mathrm{H}_{2} \mathrm{O}, 8: 2\right)$ to yield seven sub-fractions (Fr. SC14-1-SC14-7). Fr. SC14-3 $(0.12 \mathrm{~g})$ was chromatographed on a RP C-18 column $(\mathrm{MeOH} /$ $\mathrm{H}_{2} \mathrm{O}, 3: 2$ ) to give compound 2 (5.3 mg). Fr. SC14-6 (0.36 g) was chromatographed on a RP C- 18 column $\left(\mathrm{MeOH} / \mathrm{H}_{2} \mathrm{O}, 3\right.$ : 2) to give compounds 5 (76.7 mg), 6 (5.3 mg), 7 (18.3 mg), and 8 (43.3 mg).

erythro-Saucerneol (1): Colorless powder, $\mathrm{mp} 85-86^{\circ} \mathrm{C} .[\alpha]_{\mathrm{D}}^{25}$ -18 (c 0.1, MeOH). UV $\lambda_{\max }(\mathrm{MeOH}) \mathrm{nm}(\log \varepsilon): 206$ (3.74), 284 (2.94). ${ }^{1} \mathrm{H}$ - and ${ }^{13} \mathrm{C}$-NMR data see Tables 1 . HRESIMS $m / z$ 537.2471 [M-H] ${ }^{-}$(Calcd for $\mathrm{C}_{31} \mathrm{H}_{37} \mathrm{O}_{8}:$ 537.2488). CD (c $0.0004 \mathrm{MeOH}):[\theta]_{231}+12,075$.

Saucemeol J(2): Colorless powder, mp $75-76{ }^{\circ} \mathrm{C}$. $[\alpha]_{\mathrm{D}}^{25}-10$ (c $0.1, \mathrm{MeOH}) . \mathrm{UV} \lambda_{\max }(\mathrm{MeOH}) \mathrm{nm}(\log \varepsilon): 206$ (3.81), 282 (2.67). ${ }^{1} \mathrm{H}$ - and ${ }^{13} \mathrm{C}-\mathrm{NMR}$ data see Tables 1 and 2. HRESIMS $m / z 523.2310$ [M-H] ${ }^{-}$(Calcd for $\left.\mathrm{C}_{30} \mathrm{H}_{35} \mathrm{O}_{8}: 523.2332\right)$. CD (c $0.0006 \mathrm{MeOH}):[\theta]_{232}+18,121$.

Cytotoxicity evaluation. All the isolates were assessed with the HL-60 (human promyelocytic leukemia) cells according to the established protocol. $^{29}$

Acknowledgments. This research was supported by a grant of KRIBB Research Initiative Program (KGS2241012).

\section{References and Notes}

1. Chung, B. S.; Shin, M. G. Dictionary of Korean Folk Medicine; Young Lim Publishing: Seoul, 1990; pp 813-814.

2. Ahn, B. T.; Lee, S.; Lee, S. B.; Lee, E. S.; Kim, J. G.; Bok, S. H.; Jeong, T. S. J. Nat. Prod. 2001, 64, 1562.

3. Hwang, B. Y.; Lee, J. H.; Jung, H. S.; Kim, K. S.; Nam, J. B.; Hong, Y. S.; Paik, S. G.; Lee, J. J. Planta Med. 2003, 69, 1096.

4. Hwang, B. Y.; Lee, J. H.; Nam, J. B.; Hong, Y. S.; Lee, J. J. Phytochemistry 2003, 64, 765 .
5. Lee, W. S.; Baek, Y. I.; Kim, J. R.; Cho, K. H.; Sok, D. E.; Jeong, T. S. Bioorg. Med. Chem. Lett. 2004, 14, 5623.

6. Sung, S. H.; Kim, Y. C. J. Nat. Prod. 2000, 63, 1019.

7. Sung, S. H.; Lee, E. J.; Cho, J. H.; Kim, H. S.; Kim, Y. C. Biol. Pharm. Bull. 2000, 23, 666.

8. Seo, C. S.; Zheng, M. S.; Woo, M. H.; Lee, C. S.; Lee, S. H.; Jeong, B. S.; Chang, H. W.; Jahng, Y.; Lee, E. S.; Son, J. K. J. Nat. Prod. 2008, 71, 1771.

9. Sung, S. H. Fitoterapia 2006, 77, 487.

10. Kim, S. R.; Sung, S. H.; Kang, S. Y.; Koo, K. A.; Kim, S. H.; Ma, C. J.; Lee, H. S.; Park, M. J.; Kim, Y. C. Planta Med. 2004, 70, 391.

11. Kang, T. H.; Cho, H.; Oh, H.; Sohn, D. H.; Kim, Y. C. Fitoterapia 2005, 76, 115.

12. Hwang, B. Y.; Lee, J. H.; Nam, J. B.; Kim, H. S.; Hong, Y. S.; Lee, J. J. J. Nat. Prod. 2002, 65, 616.

13. Seo, B. R.; Lee, K. W.; Ha, J.; Park, H. J.; Choi, J. W.; Lee, K. T. Carcinogenesis 2004, 25, 1387.

14. Seo, B. R.; Yoo, C. B.; Park, H. J.; Choi, J. W.; Seo, K.; Choi, S. K.; Lee, K. T. Biol. Pharm. Bull. 2004, 27, 1594.

15. Song, S. Y.; Lee, I.; Park, C.; Lee, H.; Hahm, J. C.; Kang, W. K. Int. J. Mol. Med. 2005, 16, 517.

16. Hahm, J. C.; Lee, I. K.; Kang, W. K.; Kim, S. U.; Ahn, Y. J. Planta Med. 2005, 71, 464.

17. Hossain, C. F.; Kim, Y. P.; Baerson, S. R.; Zhang, L.; Bruick, R. K.; Mohammed, K. A.; Agarwal, A. K.; Nagle, D. G.; Zhou, Y. D. Biochem. Biophys. Res. Commun. 2005, 333, 1026.

18. Hodges, T. W.; Hossain, C. F.; Kim, Y. P.; Zhou, Y. D.; Nagle, D. G. J. Nat. Prod. 2004, 67, 767.

19. Hwang, B. Y.; Lee, J. H.; Koo, T. H.; Kim, H. S.; Hong, Y. S.; Ro, J. S.; Lee, K. S.; Lee, J. J. Planta Med. 2002, 68, 101.

20. Lee, A. K.; Sung, S. H.; Kim, Y. C.; Kim, S. G. Br. J. Pharmacol. 2003, 139, 11 .

21. Rho, M. C.; Kwon, O. E.; Kim, K.; Lee, S. W.; Chung, M. Y.; Kim, Y. H.; Hayashi, M.; Lee, H. S.; Kim, Y. K. Planta Med. 2003, 69, 1147.

22. Moon, T. C.; Kim, J. C.; Song, S. E.; Suh, S. J.; Seo, C. S.; Kim, Y. K.; Jin, M.; Yang, J. H.; Son, J. K.; Jahng, Y.; Kim, C. H.; Chang, H. W. Int. Immunopharmacol. 2008, 8, 1395.

23. Lee, W. S.; Lee, D. W.; Baek, Y. I.; An, S. J.; Cho, K. H.; Choi, Y. K.; Kim, H. C.; Park, H. Y.; Bae, K. H.; Jeong, T. S. Bioorg. Med. Chem. Lett. 2004, 14, 3109.

24. Park, S. Y.; Lee, S. H.; Choi, W. H.; Koh, E. M.; Seo, J. H.; Ryu, S. Y.; Kim, Y. S.; Kwon, D. Y.; Koh, W. S. Planta Med. 2007, 73, 674.

25. Hanessian, S.; Reddy, G. J.; Chahal, N. Org. Lett. 2006, 8, 5477.

26. Tofern, B.; Jenett-Siems, K.; Siems, K.; Jakupovic, J.; Eich, E.; Phytochemistry 2000, 53, 119.

27. Huo, C.; Liang, H.; Zhao, Y.; Wang, B.; Zhang, Q. Phytochemistry 2008, 69, 788 .

28. Park, H. J.; Kim, R. G.; Seo, B. R.; Ha, J.; Ahn, B. T.; Bok, S. H.; Lee, Y. S.; Kim, H. J.; Lee, K. T. Planta Med. 2003, 69, 947.

29. Park, B. Y.; Oh, S. R.; Ahn, K. S.; Kwon, O. K.; Lee, H. K. Int. Immunopharmcol. 2008, 8, 967. 